\title{
Response strategies in the radial arm maze: Running around in circles
}

\author{
SONJA I. YOERG and ALAN C. KAMIL \\ University of Massachusetts, Amherst, Massachusetts
}

\begin{abstract}
The effects of the size of the central arena on the use of response strategies by rats on an eight-arm elevated maze were examined. The size of the central arena had no effect on accuracy, but the use of adjacent arms increased significantly with a larger central arena, regardless of the size of arena to which rats were first exposed. These results are interpreted in terms of foraging efficiency.
\end{abstract}

Many experiments employing the eight-arm radial maze have shown that rats readily learn to make their choices so as to avoid arms already visited (see Olton, Handelmann, \& Walker, 1981, for a review). Virtually errorless asymptotic performance often occurs within 20 trials (Olton \& Samuelson, 1976). It has been demonstrated that neither intramaze cues nor odor trails direct choice behavior on the maze, indicating that it is the characteristics from the wider environment (i.e., the extramaze cues) that support performance (Olton \& Collison, 1979; Olton, Collison, \& Werz, 1977; Olton \& Samuelson, 1976).

The reason rats perform so accurately in the radial maze may be that this method successfully mimics certain foraging situations in the wild. Contrary to the general assumption of most psychologists, that reinforcing an animal will increase the probability of that animal's returning to the place where reinforcement occurred, the behavior of rats on the radial maze demonstrates just the opposite (Olton, 1981). This finding is less of an anomaly if placed in an ecological context. A predator whose food requires time to replete must have an accurate memory for the food locations already visited and not return to those emptied locations, if it is to forage efficiently (Gill \& Wolf, 1977; Kamil, 1978).

One rather curious finding in most radial maze studies is that the rats do not appear to use strategies. That is, there is no evidence for consistent response chains or algorithms (e.g., Olton \& Samuelson, 1976), although subjects do show a small tendency to turn in one direction or another upon leaving an arm and usually skip at least one arm before entering the next. Olton et al. (1977) conducted a more rigorous test of the importance of response

Thanks are due Russ Church, Dave Eckermann, and Dave Olton for comments and suggestions. This research was supported in part by funds received from the University of Massachusetts Psychology Department. Reprint requests should be addressed to the senior author at the Department of Psychology, University of California, Berkeley, California 94720. strategies. On a 17 -arm radial maze, rats were confined to the central arena for $20 \mathrm{sec}$ after each choice. While in the free choice condition the distribution of turn magnitudes was highly skewed in the direction of the smaller magnitudes, the confinement procedure was sufficient to disrupt any response strategies. Accuracy was unimpaired by the manipulation.

These results contradict what intuition would inform us, namely, that the simplest method of obtaining all the food available on the maze is to choose only adjacent arms. It has been pointed out that such a "choice strategy could be stored in longterm memory, and the arms could be responded to as they appeared and would not have to be individually identified or remembered"' (Olton \& Samuelson, 1976 , p. 112). A question which might be posed is whether there are conditions under which such strategies might be expected to develop.

Roberts and Dale (1981) tested rats on an eightarm maze for five massed trials per day. The results indicated that, across days and across trials within days, the percentage of choices of adjacent arms increased to over $90 \%$ for all rats. Since accuracy improved on Trials 4 and 5 (those most characterized by the adjacent arm strategy), Roberts and Dale suggest that the adoption of the strategy served to minimize proactive interference.

The physical features of the radial maze itself may also affect the pattern of responding in the maze. Optimal foraging theory predicts that animals will use foraging strategies that tend to maximize rate of food intake over a given period of time (Charnov, 1976; MacArthur \& Pianka, 1966; Pyke, Pulliam, \& Charnov, 1977). Field and laboratory research based on this prediction has yielded positive results (e.g., Krebs, Ryan, \& Charnov, 1974; Werner \& Hall, 1974). One way of maximizing rate of intake is minimizing the time (and/or energy) expended in traveling from one food source to another. Menzel (1973) demonstrated this phenomenon in chimpanzees, and discovered that the ani- 
mals retrieved hidden food not in the order in which it had been hidden, but, rather, by using a "leastdistance" or "nearest-neighbor" type of strategy. Such a strategy undoubtedly reduced the time and energy spent in traveling between cache sites, thus increasing rate of intake.

In the case of the radial arm maze, the difference in energetic cost between traveling to an adjacent arm or traveling to an opposite arm is probably negligible, given the small size of the central arena. Most of the distance traveled is incurred on the arms. However, if the size of the central arena relative to the length of the arm were to be increased, strategies involving the choice of adjacent arms might be expected to develop. The present experiment sought to determine the effect of increasing the size of the central arena of the radial maze on the pattern of selection of arms still containing food.

\section{METHOD}

\section{Subjects}

The subjects were 10 male Holtzman rats, approximately 150 days old at the start of the experiment. Some of the rats had received up to $121-\mathrm{mA}$ shocks during equipment testing unrelated to the present study. The subjects were housed individually and were maintained at $85 \%$ free-feeding weight during the course of the experiment. Daily feeding took place within $1 \mathrm{~h}$ after testing.

\section{Apparatus}

The apparatus was an eight-arm elevated radial maze modified from that of Olton and Samuelson (1976). The arms were $131 \mathrm{~cm}$ long and $8.5 \mathrm{~cm}$ wide. The proximal end of each arm was nailed to a central supporting column which stood $53 \mathrm{~cm}$ above the floor. One of two octagonally shaped arenas constructed of 3-mm-thick Masonite rested on the supporting column. The large arena was $88 \mathrm{~cm}$ in diameter, while the small arena was $34 \mathrm{~cm}$ in diameter. A block of wood, $20 \mathrm{~cm}$ high, was attached to each arm. The placement of these barriers was adjusted so that the distance from the edge of both the small and large arenas to the barrier was always $88 \mathrm{~cm}$. A plastic furniture coaster, $4.5 \mathrm{~cm}$ in diameter, was placed in front of each block and served as a food cup. The lip of the cup was $1 \mathrm{~cm}$ high and prevented visual detection of food by the rat at a distance of more than $10 \mathrm{~cm}$. The maze had no walls.

The maze was located in a large classroom, illuminated by overhead fluorescent lights. Desks, chairs, shelves, two doors, and a blackboard were present, providing abundant visual cues. An experimenter was seated in a corner of the testing room during all sessions. A manual timer with a sweep second hand was used to time trials.

\section{Procedure}

During the first 3 days, all rats were allowed to explore the apparatus with the small arena for $10 \mathrm{~min}$ each day. The subjects were then assigned randomly to either Group S-L (small arena first) or Group L-S (large arena first). The rats in each group were then given an additional 10-min session of adaptation with the arena on which they would be tested during Condition 1 . No food was present on the maze during adaptation.

For the next 27 days, Group S-L was tested with the small arena in place and Group L-S was tested with the large arena in place. The rats were given one trial per day, 7 days per week. The time to completion of the task and the sequence of arms visited were recorded. Prior to each test session, three 45-mg Noyes precision food pellets were placed in the food cup at the end of each arm. The rat was then placed in the center of the platform and a timer was started. The session ended when the rat had either obtained all the food or made 16 choices, or when $10 \mathrm{~min}$ had elapsed. A choice was defined as having occurred when the rat had placed all four feet upon an arm.

For the next 10 days, the rats in Group S-L were placed on the large arena maze, while those in Group L-S were placed on the small arena maze. The criteria for the termination of a trial were the same as for Condition 1.

\section{RESULTS}

There were no group differences in the accuracy of choice of unvisited arms during either phase of the experiment.

Both groups learned the task rapidly. By Day 8 of Condition 1, all rats had cleared the maze within 10 min during three consecutive sessions. Group S-L made a mean of 8.3 errors over these 8 days, while Group L-S made a mean of 7.8 errors $[F(1,8)<1]$.

The groups also did not differ in asymptotic accuracy. For the last 10 days before transfer (Days 1827 of Condition 1), the mean number of different arms chosen within the first eight choices was 7.9 for Group S-L and 7.7 for Group L-S $[F(1,8)=$ $1.98, p>.20$ ]. The mean number of arms chosen in order to clear the maze for these same 10 days was 8.1 for Group S-L and $\mathbf{8 . 4}$ for Group L-S $[\mathrm{F}(1,8)=1.56, \mathrm{p}>.25]$.

After transfer, both groups continued to perform accurately. The rats in Group S-L chose an average of 7.8 novel arms during the 10 days of this condition, while the rats in Group L-S chose an average of 7.7 novel arms $[F(1,8)<1]$. There were also no group differences in posttransfer accuracy in terms of the number of choices (8.4 in each group) required to complete the maze $[F(1,8)<1]$.

To demonstrate that choice accuracy was not disrupted by the transfer of Group S-L to the large maze or of Group L-S to the small maze, a $2 \times 2$ analysis of variance was conducted with group and maze type as the independent variables. The differences between groups in the number of novel arms chosen in the first eight choices before and after transfer were nonsignificant $[F(1,8)=1.11, p>.30]$. There was no effect for maze type $[F(1,8)<1]$ and no significant interaction between maze type and group $[F(1,8)=1.70, p>.20]$.

Data on the elapsed time per trial were recorded to the nearest minute. Over the last 10 days of Condition 1 and the 10 days of Condition 2, 31.0\% of all trials were completed in $1 \mathrm{~min}, 54.5 \%$ were completed in $2 \mathrm{~min}, 10.0 \%$ were completed in $3 \mathrm{~min}$, and $4.5 \%$ were completed in $4 \mathrm{~min}$ or more. Inspection of the mean elapsed time per group per condition revealed no consistent difference in the time 
required to clear the large and small mazes. Group S-L required means of 1.9 min per trial in Condition 1 and $2.4 \mathrm{~min}$ per trial in Condition 2. Group L-S completed the task in means of 1.7 and $1.6 \mathrm{~min}$ per trial in Conditions 1 and 2, respectively. Generally, the rats spent little time in the central area, making their choices quickly.

If the rats on the large maze were employing a strategy involving adjacent arms, then we would expect to find significantly more turn magnitudes of \pm 1 (e.g., a choice of arm 5, followed by a choice of either arm 4 or arm 6) for these rats, regardless of whether they ran on the large maze before or after transfer. This expected result was found. The percentage of \pm 1 turn magnitudes for Group S-L was $35.3 \%$ on the small maze and increased to $69.1 \%$ on the large maze. Group L-S had a mean of $56.6 \%$ \pm 1 turn magnitudes on the large maze. The mean percentage fell to $38.1 \%$ when these rats were transferred to the small maze. The percentage of \pm 1 . turn magnitudes on the large maze was greater than that on the small maze, irrespective of group $[F(1,8)$ $=18.02, \mathrm{p}<.01$ ] (see Figure 1). The mean percentage of \pm 1 turn magnitudes across groups on the large maze was $62.8 \%$, and was as high as $97.1 \%$ for two of the rats in Group L-S. For the small maze, the mean percentage of \pm 1 turn magnitudes across groups was $36.7 \%$. There was no main effect for group $[F(1,8)<1]$ and no interaction between group and maze type $[F(1,8)=1.52, p>.20]$.

A similar analysis of the relative frequency of \pm 2 turn magnitudes indicated that the rats made signif-

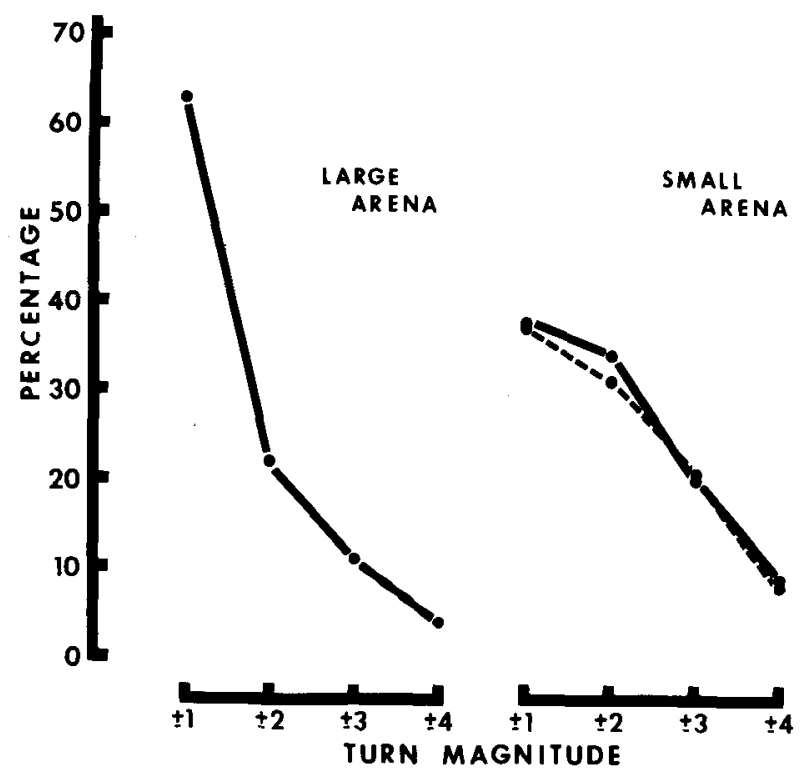

Figure 1. The distribution of turn magnitudes on the large and small mazes, collapsed over groups. The dotted line on the right-hand panel is the mean distribution from the control animals in Experiments 1 and 2 (Eckermann et al., 1980). icantly more turns of this magnitude when on the small maze, irrespective of group $[F(1,8)=8.35$, $p<.05$ ], although the effect was not as large as that for turn magnitudes of \pm 1 (see Figure 1). The mean percentage of \pm 2 turn magnitudes across groups on the small maze was $33.9 \%$, with the highest percentage of any individual rat being $60.3 \%$. For the large maze, the mean percentage of \pm 2 turn magnitudes across groups was $21.3 \%$. No main effect for groups was found $[F(1,8)<1]$, and there was no interaction between group and maze type $[F(1,8)=3.30, p>.10]$. The distribution of all turn magnitudes for pre- and posttransfer trials on small and large mazes is presented in Figure 1. Sample data from two rats in each group are given in the Appendix.

\section{DISCUSSION}

Increasing the size of the central arena from 34 to $88 \mathrm{~cm}$ resulted in a distribution of turn magnitudes highly skewed in the direction of adjacent arms. This effect was evident regardless of the order of presentation of the large and small arenas, demonstrating that previous experience on the small maze neither interfered with nor was necessary for the observed change in response patterning. Furthermore, after 27 days of experience on the large maze, the percentage of \pm 1 turn magnitudes of Group L-S during only 10 days of testing on the small maze was not different from that of Group S-L on the small maze. The behavior of Group S-L also changed rapidly when tested in the large maze; the percentage of \pm 1 turn magnitudes increased to the level exhibited by Group L-S at the end of Condition 1 .

These results strongly suggest that increasing the cost of choosing nonadjacent arms by enlarging the central arena is sufficient to produce nonrandom choice sequences. However, the high degree of accuracy obtained on both mazes, with or without the use of the adjacent arm response strategy, shows that mnemonic ability and strategy use were independent dimensions of performance in this experiment.

It might be instructive to compare the data obtained from the small maze with those of other experiments using similar apparatus and procedure. In terms of accuracy, the performance of our subjects was as good as or better than that reported for rats in similar situations (e.g., Olton \& Samuelson, 1976). Furthermore, the distribution of turn magnitudes reported by Eckermann, Gordon, Edwards, MacPhail, and Gage (1980) in their nondrug groups of rats is highly comparable to the distribution obtained from small maze trials in the present study (see Figure 1). It is therefore likely that the results presented here are general. 
Bond (Note 1) and Olton (Note 2) have suggested that the increased use of adjacent arms may be the result of the larger turning arc between adjacent arms in the large maze. For example, rats in the small maze may not be able to easily negotiate the relatively sharp angle required to enter a neighboring arm. There may be some optimal turning radius (possibly determined by rat size and a mechanical advantage of maintaining constant velocity) that combined with distance traveled to affect the distribution of turn magnitudes. These ideas are not inconsistent with our results, particularly the relatively frequent use of \pm 2 turn magnitudes in the small maze.

Some rather weak evidence against these notions comes from the behavior of drugged rats on the radial maze (Eckermann et al., 1980). One might expect difficulty in making a turn to be a function of the velocity of the animal. In comparison with saline control animals, amphetamine-injected rats cleared the maze more quickly, although their accuracy and pattern of arm selection remained unaffected. If these rats were making more choices per minute because they were running faster, then this would appear to be inconsistent with the notion that the choice of nonadjacent arms on the small maze is due to some optimal or convenient turning angle. It seems certain that the choice behavior of rats on the radial maze is affected by an entire set of variables, such as distance traveled and turning angle, which have received little attention.

This experiment tested a common sense hypothesis. Imagine a radial arm maze with a central arena $1 \mathrm{~km}$ in diameter. The gains in efficiency which an adjacent arm strategy would confer are obvious. Nonetheless, the phenomenon reported here suggests several intriguing possibilities for future research, including the role of the ratio of arm length to arena size and the influence of response strategies on memory when confinement procedures are used.

\section{REFERENCE NOTES}

1. Bond, A. B. Personal communication, 1982.

2. Olton, D. S. Personal communication, 1982.

\section{REFERENCES}

Charnov, E. L. Optimal foraging: The marginal value theorem. Theoretical Population Biology, 1976, 2, 129-136.

Eckermann, D.A., Gordon, W. A., Edwards, J.D., MacPhall, R. C., \& GAGE, M. I. Effects of scopolamine, pentobarbital, and amphetamine on radial arm maze performance in the rat. Pharmacology, Biochemistry, and Behavior, 1980, 12, 595-602.

GILL, F. R., \& WoLF, L. L. Non-random foraging by sunbirds in a patchy environment. Ecology, 1977, 58, 1284-1296.

Kamil, A. C. Systematic foraging by a nectar-feeding bird, the Amakihi (Loxops virens). Journal of Comparative and Physiological Psychology, 1978, 92, 388-396.

Krebs, J. R., Ryan, J. C., \& Charnov, E. L. Hunting by expectation or optimal foraging? A study of patch use by chickadees. Animal Behaviour, 1974, 22, 953-964.

MacArthur, R. H., \& Pianka, E. R. On the optimal use of a patchy environment. American Naturalist, 1966, 100, 603-609.

MENzeL, E. W. Chimpanzee spatial memory organization. Science, 1973, 182, 943-945.

Olton, D. S., \& Collison, C. Intramaze cues and "odor trails" fail to direct choice behavior on an elevated maze. Animal Learning \& Behavior, 1979, 7, 221-223.

Olton, D. S., Collison, C., \& Werz, M. A. Spatial memory and radial arm maze performance in rats. Learning and Motivation, 1977, 8, 289-314.

Olton, D. S., Handelmann, G. E., \& Walker, J. A. Spatial memory and food searching strategies. In A. C. Kamil \& T. D. Sargent (Eds.), Foraging behavior: Ecological, ethological, and psychological approaches. New York: Garland, 1981.

Olton, D. S., \& SAmuelson, R. J. Remembrance of places passed: Spatial memory in rats. Journal of Experimental Psychology: Animal Behavior Processes, 1976, 2, 97-116.

Pyke, G. H., Pulliam, H. R., \& Charnov, E. L. Optimal foraging: A selective review of theory and tests. Quarterly $R e$ view of Biology, 1977, 52, 137-154.

Roberts, W. A., \& DALE, R. H. I. Remembrance of places lasts: Proactive inhibition and patterns of choice in rat spatial memory. Learning and Motivation, 1981, 12, 261-281.

Werner, E. E., \& Hall, D. J. Optimal foraging and the size selection of prey by the bluegill sunfish (Lepomis macrochirus). Ecology, 1974, 55, 1042-1052.

Appendix

Raw Data From Two Rats From Group S-L and Two Rats From Group L-S During the Last 10 Days of Condition 1 and the First 10 Days of Condition 2

\begin{tabular}{|c|c|c|c|c|c|c|c|c|c|c|c|c|c|c|c|c|c|c|c|c|c|c|c|c|c|c|c|c|c|c|}
\hline \multicolumn{16}{|c|}{ Group S-L } & \multicolumn{15}{|c|}{ Group L-S } \\
\hline \multicolumn{8}{|c|}{ Small Maze } & \multicolumn{8}{|c|}{ Large Maze } & \multicolumn{7}{|c|}{ Large Maze } & \multicolumn{8}{|c|}{ Small Maze } \\
\hline \multicolumn{16}{|c|}{$\mathrm{R} 2$} & & & & & & & & R8 & & & & & & & \\
\hline 3 & 8 & 5 & 6 & 4 & 1 & 7 & & 7 & 6 & 8 & 3 & 1 & 5 & 4 & & 8 & 7 & 65 & 54 & 3 & 2 & 1 & 8 & 7 & 6 & 5 & 4 & 3 & 2 & \\
\hline 6 & 8 & 4 & 1 & 7 & 5 & 3 & & 8 & 7 & 1 & 2 & 3 & 5 & 6 & & 8 & 7 & 65 & 54 & 3 & 2 & 1 & 8 & 7 & 6 & 5 & 4 & 3 & 21 & \\
\hline 5 & 3 & 1 & 4 & 8 & 2 & 7 & & 2 & 5 & 6 & 7 & 8 & 3 & 4 & & 8 & 7 & 65 & 54 & 3 & 2 & 1 & 7 & 6 & 5 & 4 & 3 & 2 & 18 & 8 \\
\hline 3 & 6 & 1 & 2 & 8 & 5 & 7 & & 8 & 1 & 3 & 4 & 5 & 7 & 2 & & 8 & 7 & 65 & 54 & 3 & 2 & 1 & 8 & 7 & 6 & 5 & 4 & 3 & 21 & \\
\hline 8 & 6 & 4 & 7 & 5 & 3 & 1 & & 3 & 6 & 7 & 8 & 2 & 5 & 1 & & 8 & 7 & 6 & 54 & 3 & 2 & 1 & 5 & 6 & 7 & 8 & 1 & 2 & 4 & \\
\hline 8 & 5 & 6 & 2 & 7 & 4 & 1 & 3 & 1 & 4 & 5 & 7 & 8 & 3 & 1 & 6 & 8 & 7 & 6 & 54 & 3 & 2 & 1 & 1 & 2 & 3 & 4 & 5 & 6 & 7 & \\
\hline 4 & 2 & 8 & 5 & 3 & 1 & 6 & & 7 & 5 & 4 & 3 & 1 & 8 & 2 & & 8 & 7 & 65 & 54 & 3 & 2 & 1 & 1 & 2 & 3 & 4 & 5 & 6 & 7 & \\
\hline 2 & 8 & 5 & 4 & 3 & 1 & 6 & & 8 & 3 & 2 & 6 & 4 & 1 & 5 & & 8 & 7 & 65 & 54 & 3 & 2 & 1 & 1 & 2 & 3 & 4 & 5 & 6 & 78 & 8 \\
\hline 7 & 1 & 5 & 8 & 6 & 4 & 3 & & 1 & 4 & 5 & 7 & 8 & 3 & 1 & 6 & 1 & 3 & 4 & 56 & 7 & 8 & 2 & 8 & 7 & 6 & & & 3 & 2 & \\
\hline 8 & 6 & 14 & 42 & 7 & 8 & 3 & 45 & 1 & 7 & 6 & 5 & 4 & 32 & 8 & & 1 & 2 & 34 & 45 & 6 & 7 & 8 & 6 & 5 & 4 & 3 & 21 & 18 & 87 & 7 \\
\hline
\end{tabular}


Group S-L

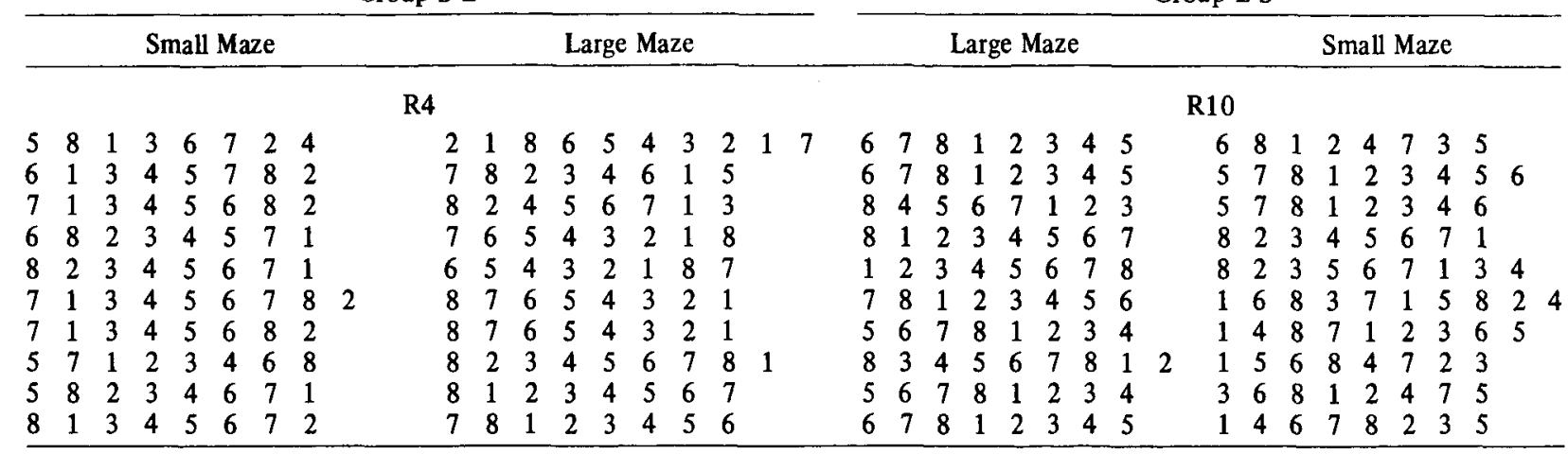

Note-R4 and R10 were chosen because their choice patterning was clearly dependent upon the size of maze used. $R 2$ and $R 8$ were the rats whose behavior was the least responsive to the manipulation. Numbers 18 refer to the arms of the radial maze.
(Manuscript received February 8, 1982;

revision accepted for publication May 21, 1982.) 